\title{
Smart Communication for Cooperative Wireless Sensor Network
}

\author{
A. Belbachir ${ }^{1}$ and S. Benabid ${ }^{1}$
}

\begin{abstract}
Several embedded systems use Wireless Sensor Network (WSN) to monitor an area. However, efficient robust and reliable communication between sensors is hard to achieve. Thus, in this paper we focus on exploration area and we propose a new cooperative strategy. This strategy is based on Cognitive Radio (CR) and Software Defined Radio (SDR) that we consider as a "Smart Communication". These radio systems search for a vacant spectrum band and reconfigures itself satisfying the requirements of any desired communication standard. Several simulation experiments demonstrate that the proposed approach improve exploration strategy.
\end{abstract}

Index Terms-Cognitive Radio, Software Defined Radio, Wireless Sensor Network, Exploration Strategy.

\section{INTRODUCTION}

Recent technological advances pushed Wireless Sensor Networks (WSNs) to gain a big place in our daily lives. From intelligent transport [1] to healthcare applications [2], WSNs are everywhere. WSNs contains several sensor nodes, that are usually used in an area of deployment and coordinate themselves to give a good information quality about the physical environment. Each sensor node bases its decisions on its own collected information. Then, these sensors communicate either among each other or to an external base-station. To send these information, each node should communicate on several radio standards, with different carrier frequencies, bandwidths and data rates. Integrating all those standards in one node would require a dedicated radio front-end for each standard. This would significantly increase the cost and area of the device. In this context, realizing a reconfigurable and programmable radio front-end able to meet the requirements of any desired communication standard while maintaining a consistent performance has became a necessity. For this reason, we propose to use the Cognitive Radio (CR) and Software Defined Radio (SDR) concept as shown in Fig. 1.

A CR is an intelligent radio capable to obtain the knowledge of radio operational environment and to dynamically adjust its operational parameters and protocols [3]. In other words, CR can sense the communication environment and detect spectrum hole to improve the communication efficiency [4]. SDR is considered a technology enabler for CR, which can adapt their transmission/reception frequencies and parameters. Therefore, the main challenge in designing a SDR is to develop an efficient procedure to reconfigure instantly the radio system to the appropriate signal format.

1 Polytechnic Institute of Advanced Sciences, Ivry-sur-Seine, France. email: assia.belbachir@ipsa.fr, sorore.benabid@ipsa.fr

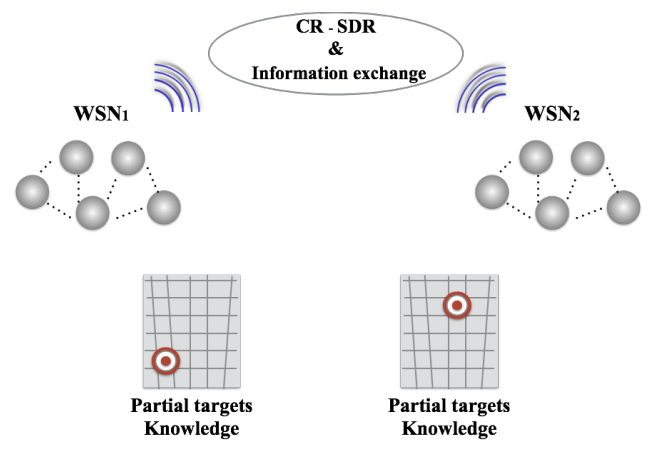

Fig. 1: WSN Cooperation for targets localization based on $\mathrm{CR}$ and SDR.

Additionally, the WSNs suffer from low quality of sensor nodes and their short lifetime. One strategy to reduce these disadvantages is the use of diversity [5] [6] and share information coming from other sensor nodes to provide more faithful information.

In our case of study, we consider a set of sensor nodes as a robot. Each robot can communicate with the robots that are in their range of coverage. These robots accomplish an exploration mission of an unknown area (e.g. forest [7]) to detect anomalies in the environment such as forest fire. We are interested to explore an area to localize the maximal number of targets with a reduced exploration time. We used an innovative cooperative exploration strategy to help the robots to optimize the exploration strategy using CR and SDR technology.

In this paper, we study the impact of the cooperative exploration strategy in WSN based on smart communication platform. We report, in section II, different exploration strategies. Section III describes the principle of CR and SDR technology, then the multi-standard transceiver. Section IV explains the target and sensor model, then the developed exploration strategy. The obtained simulation results are shown and discussed in section V. Finally, we conclude our paper.

\section{BACKGROUND}

Exploration represents the task of guiding the system to cover its environment using the information coming from its own sensors or its neighbors. Efficient exploration strategies are useful when the area to explore is unknown and that we can't explore the whole area with a reduced exploration time. Several research has been focussing in the problem of simultaneous localization and mapping [8], an aspect that we do not address in this paper.

A usual exploration strategy is to extract frontiers 
TABLE I: Standard Specifications for WSNs

\begin{tabular}{|c|c|c|c|l|l|}
\hline Standard & GSM900/GSM1900 & WCDMA & Zigbee & Bluetooth & IEEE802.11a/g \\
\hline Operating Spectrum & $890-915 \mathrm{MHz} / 1930-1980 \mathrm{MHz}$ & $1920-1980 \mathrm{MHz}$ & $2.4 \mathrm{GHz}$ & $\begin{array}{l}2.4-2.4835 \\
\mathrm{GHz}\end{array}$ & $5 \mathrm{GHz} / 2.4 \mathrm{GHz}$ \\
\hline Data Rate & $271 \mathrm{~Kb} / \mathrm{s}$ & $384 \mathrm{~Kb} / \mathrm{s}$ & $250 \mathrm{~Kb} / \mathrm{s}$ & $1 \mathrm{Mb} / \mathrm{s}$ & $\mathrm{Up} \mathrm{to} 54 \mathrm{Mb} / \mathrm{s}$ \\
\hline Channel BW & $200 \mathrm{KHz} / 1.23 \mathrm{MHz}$ & $5 \mathrm{MHz}$ & $2 \mathrm{MHz}$ & $1 \mathrm{MHz}$ & $20 \mathrm{MHz}$ \\
\hline Peak Power & $33 \mathrm{dBm} / 30 \mathrm{dBm}$ & $-49 \mathrm{dBm}$ to $24 \mathrm{dBm}$ & $0 \mathrm{dBm}, 18 \mathrm{dBm}$ & $0,4,20 \mathrm{dBm}$ & $\begin{array}{l}7 \mathrm{do} 20 \mathrm{dBm}(11 . \mathrm{a}) \\
0 \text { to } 20 \mathrm{dBm}(11 . \mathrm{g})\end{array}$ \\
\hline
\end{tabular}

between known and unknown areas [9] [10] and to visit the closest unexplored area. These approaches only distinguish between explored and un-explored areas and do not take into account the gathered information at each system or robot. Thus, these approaches can't deal with targets localization. To overcome this limitation we propose to introduce information related to the system perception and its neighbors.

\section{Smart COMmunication Plateforme}

\section{A. CR and SDR technology}

In the past decade, CR and SDR technology has revolutionized our view of opportunities in wireless communications to a great extent. The design and deployment of this technology has been investigated in a number of papers and research studies starting from the papers of Joseph Mitola [3] [4].

The key motivation of the CR technology is to increase spectral utilization and to optimize the use of radio resources. As illustrated in Fig. 2, the CR determine which portions of the spectrum are available, detect the presence of primary users and select the best available channel for communication [11].

The successful deployment of CR technologie will depend on the design and implementation of an efficient SDR platforme. A simple approach to realize this platform is to implement several radio front-end circuits in parallel. However, it leads to large area and high cost. Therefore, the solution is to implement a radio front-end by a number of reconfigurable circuits operating in a number of frequency bands. The frequency bands can easily be changed without modifying the hardware, giving WSN considerable flexibility.

\section{B. SDR Transceiver}

The main requirement of an SDR transceiver is to be able to support simultaneously multiple standards (GSM, Wifi, Bluetooth, Zigbee, etc.) covering from $0.8 \mathrm{GHz}$ to $5 \mathrm{GHz}$ frequency bands, without any need to change the hardware of the radio. The SDR transceiver is shown in Fig. 3 in which the major part of the transmitter (TX) path and receiver (RX) path is implemented by means of digital device instead of analog one as in conventional transceiver. The digital

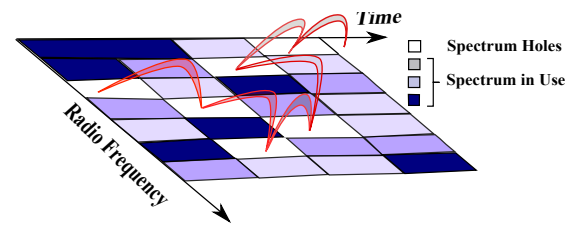

Fig. 2: CR Concept.

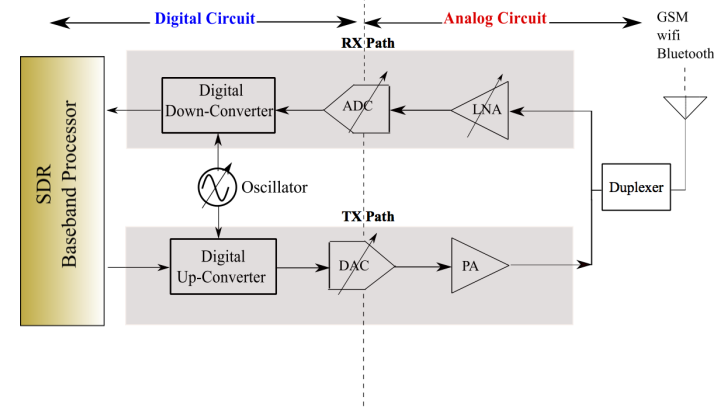

Fig. 3: Gereric SDR Transceiver

implementation provides a higher degree of flexibility and reconfigurability. For this reason, an analog-todigital converter (ADC) and digital-to-analog converter (DAC) take place near to the antenna [12] [13].

In the analog part of the transceiver, we use a duplexer which selects the required bands (to and from the antenna) and separates the corresponding transmits and receives sections. A variable Low Noise Amplifier (LNA) performs the initial amplification of the received signals. The transmitters employ a carefully designed power amplifier (PA) to deliver the required power to the antenna.

The software base-band section performs base-band operations (e.g. frequency hopping), including the capability to change the channel assignments and to modify the transmission parameters or communication protocols. The software device include field programmable gate array (FPGA), digital signal processors (DSP) and programmable system on chip (SoC).

We reporte in Table I, the specifications of the main standards that could potentially be used for a WSN.

\section{Models And Method FOR EXPLORATION}

In this section, we explain the developed exploration strategy that is used to maximize the number of found targets. This new exploration strategy deals also with the communication standard that is changing with time (using CR-SDR). We show as follow our target and sensor model, the discret reasoning environment and our developed exploration strategy.

\section{A. Target and Sensor Model}

We simplify our model and consider the model as stationary target and not time dependent. The emission of the target expands as it raises higher to the atmosphere. In our model, we defined a target model, according to the maximal temperature as known $\left(T_{M A X}\right)$.

The temperature density $T$ within the target is a decreasing function of the horizontal distance $\rho$ with 
respect to the forest fire center and the elevation $z$ above the atmosphere (Fig. 4).

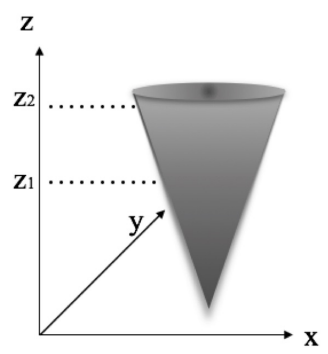

Fig. 4: Illustration of the temperature evolution within a target in stationary weather: the temperature, here represented in red, decreases with the elevation and with the distance to the vertical of the emitting fire.

This function is the model of the forest on fire, which is an approximation of the actual diffusion phenomenon: the model is probabilistic and expresses the probability density function ( $p d f$ ) of the temperature $T$ as a function of the distance $\rho$ and the elevation $z$ :

$$
P(T=t \mid \rho, z)
$$

Fig. 5 shows the behavior of the model at two different levels: the dispersion of the temperature, represented by a dashed lines, is also an increasing function of the distance and the high.

The used sensor is a range-only sensor. This type of sensor perceives the distance to the target with an error. When $T_{M A X}$ of the target is known, two temperature measures provide an estimate of the distance to the target.

For a given position $(\rho, z)$ in the target, the probabilistic variations of the temperature is modeled by a Gaussian: $T=\mathbb{N}\left(T_{\text {mean }}, \rho(\tau, z)\right)$, where $\sigma(\rho, z)$ is an increasing function of $\rho$ and $\mathrm{z}$. We can model temperature sensors with a probability density function $P\left(T_{\text {sensor }} \mid T\right)$ that models its errors such as a Gaussian.

Fig. 6 represents two maps. The map in the top shows the target position and two displacements of the robot with several sensors. The second map represents the computed probability for the robot with several sensors.

The distance is estimated with an error that increase with the distance from the target direction. The model is defined by the probability density function of the temperature $T$ as a function of the distance $\rho$ and the elevation $z$ (defined in (1)).

\section{B. Probabilistic Card and Exploration Strategy}

We choose to discretize our environment into a grid. For each grid square, a probability that the cell contains a target [14] is associated. Additionally, we added two other information in the same cell related to connectivity and exploration. Connectivity information represents the ratio between the number of nodes in the communication range of the robot and the total number of the nodes in the network. Exploration information is equal to " 1 " if the cell was already explored by the robot, otherwise it is equal to " 0 ". The cell probabilities

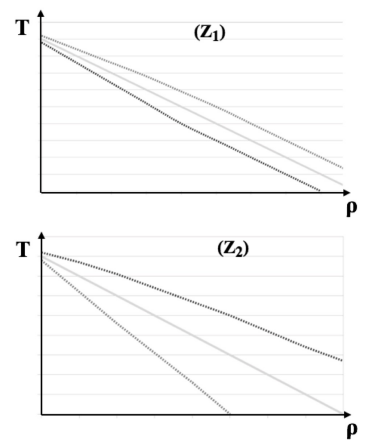

Fig. 5: Evolution of the temperature as a function of the horizontal distance $\rho$ for two depths $z_{1}$ and $z_{2}$ defined in Fig.4.

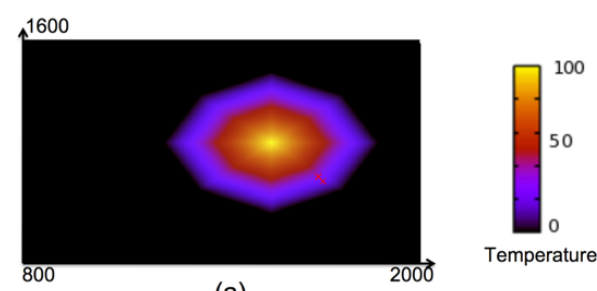

(a)

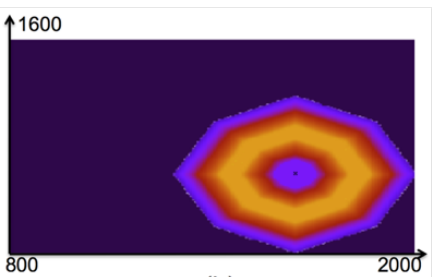

(b)

Fig. 6: Illustration of the target model at a given depth. The colors indicate the temperature evolution in the figure (a) and the probability of the target presence on a regular Cartesian grid in the figure (b).

are updated incrementally according to a classical bayesian paradigm under a markovian assumption:

$$
P^{k}\left(x_{i, j}\right)=\frac{P\left(T^{k} \mid x_{i, j}=\text { target }\right) P^{k-1}\left(x_{i, j}\right)}{P\left(T^{k}\right)}
$$

where $P\left(T^{k} \mid x_{i, j}=\right.$ target $)$ is the sensor model and $P^{k-1}$ is probability value of the target existence at the time $k-1$. Note that the probability $P^{k}\left(x_{i, j}\right)$ implicitly represents the precision of the source location: a probability equal to 1 meaning that the target is perfectly localized.

Building a map is the basis on which the robot motion can be adapted in order to augment the knowledge on the environment. We defined one strategy in which the robot select the next motions, depending on a source presence hypothesis (a local maximum of $P\left(x_{i, j}\right)$ in the mapped vicinity of the current robot position). One greedy strategy aims at confirming the presence of a target, assessed when its probability exceeds a threshold $P_{\text {conf }}\left(P_{\text {conf }}<1\right)$. 
TABLE II: Statistical Results For target localization using graph connectivity.

\begin{tabular}{|c|c|c|c|c|c|}
\hline Amount of robots & Amount of targets & Exploration time (ut) & Found Targets & Graph connectivity & $\%$ found targets \\
\hline 1 & 13 & 100 & 4 & - & $0.3 \%$ \\
\hline 1 & 20 & 100 & 7 & - & $0.35 \%$ \\
\hline 3 & 10 & 100 & 7 & Yes $(100 \%)$ & $0.7 \%$ \\
\hline 3 & 10 & 100 & 8 & Yes $(85 \%)$ & $0.8 \%$ \\
\hline 3 & 10 & 100 & 7 & Yes $(50 \%)$ & $0.7 \%$ \\
\hline 3 & 10 & 100 & 7 & Yes $(25 \%)$ & $0.7 \%$ \\
\hline 4 & 30 & 100 & 15 & Yes $(100 \%)$ & $0.5 \%$ \\
\hline 4 & 30 & 100 & 21 & Yes $(85 \%)$ & $0.7 \%$ \\
\hline 4 & 30 & 100 & 20 & Yes $(50 \%)$ & $0.7 \%$ \\
\hline
\end{tabular}

\section{Simulation and evaluation Results}

In our simulation, we setup as a first experiment several wireless sensor nodes to explore an area that contains several targets (e.g. forest-fire). In the beginning, a predefined plan (such as trajectory), is embedded for each set of sensor node that represents a robot. However, with time and according to the robot perception, it modifies its own plan using its probabilistic map (see section IV) to detect a maximal number of targets. The next cell to explore is also chosen according to the connectivity. If the probability of two cells are the same, the robot is choosing the one that maintain a high connectivity of the sensor nodes. When two nodes are in the same coverage area, they can exchange their maps. Table II represents the statistical obtained result of different robots. To evaluate our exploration strategy, we used, in our first experiments, one robot. Compared to non adaptive exploration strategy, our strategy gives improvements when the time is limited to explore the whole area. Then, we implemented our exploration strategy with several robots. The obtained results in term of number of found target compared to the graph connectivity. From our experiments, we can remark that when the connectivity is $100 \%, 50 \%$ and $25 \%$, the percentage of found targets is not maximal. However, when the connectivity is $85 \%$, the percentage of found targets is maximal. We can conclude that, according to our experiments, the connectivity plays an important role to detect targets. When the connectivity is high, the system can't explore the area in an optimal way. The same remark can be applied to the system when the connectivity is low. Another conclusion is that using our cooperative exploration strategy, the localization of targets is improved than the one that are using independent robots exploration.

\section{CONCLUSION}

In this paper, we propose a new approach of cooperative Wireless Sensor Network based on Cognitive Radio (CR) and Software Defined Radio (SDR) within we have considered as smart communication. These technologies are a promising radio that increase spectrum utilization and therefore improve sensor connectivity. Based on these notions, we implemented an exploration strategy to detect targets in unknown area. Simulation results demonstrate the impact of the sensor network connectivity on the number of target localisation. For our scenario, $85 \%$ of connectivity is sufficient to improve target localization.

\section{REFERENCES}

[1] V. Katiyar, P. Kumar, and N. Chand, "Article: An intelligent transportation systems architecture using wireless sensor networks," International Journal of Computer Applications, vol. 14, no. 2, pp. 22-26, January 2011, full text available.

[2] H. Alemdar and C. Ersoy, "Wireless sensor networks for healthcare: A survey," Computer Networks, vol. 54, no. 15, pp. 2688 - 2710, 2010.

[3] J. Mitola III and G. Q. Maguire Jr, "Cognitive radio: making software radios more personal," Personal Communications, IEEE, vol. 6, no. 4, pp. 13-18, 1999.

[4] J. Mitola, "The software radio architecture," Communications Magazine, IEEE, vol. 33, no. 5, pp. 26-38, 1995.

[5] V. Tarokh, N. Seshadri, and A. R. Calderbank, "Space-time codes for high data rate wireless communication: Performance criterion and code construction," IEEE TRANS. INFORM. THEORY, vol. 44, no. 2, pp. 744-765, 1998.

[6] V. Tarokh, H. Jafarkhani, and A. Calderbank, "Space-time block codes from orthogonal designs," Information Theory, IEEE Transactions on, vol. 45, no. 5, pp. 1456-1467, Jul 1999.

[7] A. Belbachir, J. Escareno, E. Rubio, and H. Sossa, "Preliminary results on uav-based forest fire localization based on decisional navigation," in 3rd Workshop on Research, Education and Development of Unmanned Aerial Systems RED-UAS, Nov. 23252015.

[8] W. Burgard, M. Moors, and F. Schneider, Advances in PlanBased Control of Robotic Agents: International Seminar Dagstuhl Castle, Germany, October 21-26, 2001 Revised Papers. Berlin, Heidelberg: Springer Berlin Heidelberg, 2002, ch. Collaborative Exploration of Unknown Environments with Teams of Mobile Robots, pp. 52-70.

[9] C. Stachniss, Ó. Martínez Mozos, and W. Burgard, "Efficient exploration of unknown indoor environments using a team of mobile robots," Annals of Mathematics and Artificial Intelligence, vol. 52, no. 2, pp. 205-227, 2009. [Online]. Available: http://dx.doi.org/10.1007/s10472-009-9123-z

[10] B. Yamauchi, A. Schultz, and W. Adams, "Mobile robot exploration and map-building with continuous localization," in Robotics and Automation, 1998. Proceedings. 1998 IEEE International Conference on, vol. 4, May 1998, pp. 3715-3720 vol.4.

[11] I. F. Akyildiz, W.-Y. Lee, M. C. Vuran, and S. Mohanty, "Next generation/dynamic spectrum access/cognitive radio wireless networks: a survey," Computer networks, vol. 50, no. 13, pp. 2127-2159, 2006.

[12] B. Razavi, "The future of radios," in Circuits and Systems (ISCAS), 2015 IEEE International Symposium on, May 2015, pp. 1-8.

[13] S. Benabid, "Design and simulation of a 6th order continuoustime sigma-delta modulator using integrated lc filter in a standard cmos technology," in International Conference on Applied Electronics, September 2014, pp. 21-25.

[14] K. H. Low, J. Dolan, and P. Khosla, "Information-theoretic approach to efficient adaptive path planning for mobile robotic environmental sensing," in Proceedings of the 19th International Conference on Automated Planning and Scheduling (ICAPS-09), September 2009. 